\title{
Variabilité des teneurs et compositions des composés mineurs dans I'huile de tournesol au cours du développement du capitule
}

\author{
Partie II - Phytostérols
}

\author{
Alicia AYERDI-GOTOR ${ }^{1}$ \\ Monique BERGER ${ }^{1}$ \\ Françoise LABALETTE ${ }^{2}$ \\ Sylvie CENTIS ${ }^{3}$ \\ Valérie EYCHENNE ${ }^{4}$ \\ Jean DAYDÉ ${ }^{1}$ \\ Anne CALMON ${ }^{1}$ \\ ${ }^{1}$ Université de Toulouse ; École d'Ingénieurs \\ de Purpan ; Laboratoire d'Agro-Physiologie ; \\ 75, voie du TOEC, BP 57611, \\ F-31076 Toulouse Cedex 03, France \\ <anne.calmon@purpan.fr> \\ 2 ONIDOL, Organisation nationale \\ interprofessionnelle des oléagineux, \\ 12, avenue George V, 75008 Paris, France \\ ${ }^{3}$ ASEDIS-SO, 39, chemin Virebent, \\ 31200 Toulouse, France \\ ${ }^{4}$ CoGNIS-France, Usine d'Estarac, \\ 31360 Boussens, France
}

Article reçu le 12 novembre 2008

accepté le 23 décembre 2008

\begin{abstract}
Sunflower oil is rich in polyunsaturated fatty acids and in minor components (1\% of the oil fraction) including phytosterols, tocopherols, waxes and others components. This study evaluates the content and composition of the phytosterols in four sunflower hybrids during two growing seasons (2002 \& 2003) and in seven more hybrids in a multilocal study in 2003. The phytosterol content in the maturing seeds was followed from flowering stage till full maturity. The kinetics of phytosterols accumulation was similar to that of fatty acids : a rapid increase until 25 Days After Flowering DAF and a stabilization after 50 DAF. However, the temperature during the seed development induced changes in the phytosterol content, since the total amount of phytosterols was significantly increased by high temperatures in some hybrids. On the other hand, the comparison of phytosterol content in the 11 hybrids tested in 2003 shows significant differences in phytosterol content between hybrids and low location X hybrid interaction. In this study the environment effect was the major source of variation. This study shows that phytosterol content in sunflower oil can be promoted though combined action on selection and growth conditions.
\end{abstract}

Key words: sunflower oil, phytosterols, accumulation, temperature

\section{Introduction}

L'huile de tournesol bénéficie d'une bonne image en raison de sa composition en acides gras insaturés (acides oléique et linoléique) [1]. Sa faible teneur en acide linolénique $(0,2 \%)$ en fait une huile stable, classée parmi les huiles de friture, ceci d'autant plus qu'il existe maintenant des variétés contenant plus de $85 \%$ d'acide oléique, mono-insaturé [2]. Son profil en composés mineurs : richesse en tocophérols (vitamine E) et composition originale en phytostérols, apporte un intérêt nutritionnel supplémentaire [3]. L'huile de tournesol présente en outre un intérêt industriel pour la production d'esters d'acides gras (biocarburants et biolubrifiants) [4-6]. Dans ce cas, lors du processus de fabrication et plus particulièrement de désodorisation, une fraction dite « insaponifiable » contenant les composés mineurs (tocophérols et phytostérols) est isolée. Cette fraction peut être valorisée en un co-produit à forte valeur ajoutée [7].

Les phytostérols représentent une vaste famille de composés, de structure analogue à celle du cholestérol chez les animaux, et dont la fonction biologique est semblable. Ce sont des alcools stéroïdiens avec un noyau tétracyclique et une chaîne latérale de 17 atomes de carbone (figure 1). Les principaux phytostérols (60 à $80 \%$ ) trouvés dans l'huile de tournesol sont par ordre

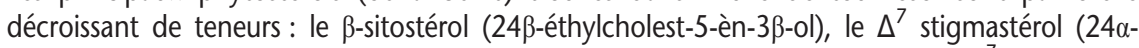

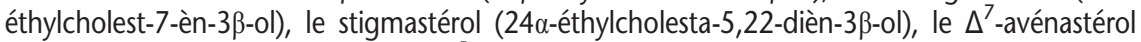
(24-éthylidenecholest-7-èn-3 $\beta$-ol), le $\Delta^{5}$-avénastérol (24-éthylidencholest-5-èn-3 $\beta$-ol), le campes-

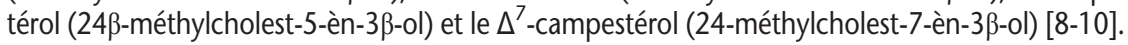
Les phytostérols permettent de rigidifier et de contrôler le niveau de fluidité des membranes cellulaires. Mais leur rôle serait plus actif qu'on ne le pensait initialement : leur implication dans les régulations hormonales est très étudiée, aussi bien en tant que précurseurs des brassinostéroïdes que directement au niveau de l'organisation de la membrane cellulaire, où ils seraient d'importants médiateurs de l'interaction entre les protéines et les lipides membranaires [11, 12]. Les effets des phytostérols sur la santé humaine font l'objet de nombreux travaux depuis quelques années [13-16]. C'est en raison de leur pouvoir hypocholestérolémiant que les stérols d'origine végétale ont suscité le plus d'intérêt. Grâce à leurs propriétés amphiphiles, ils inhibent l'absorption intesti- 


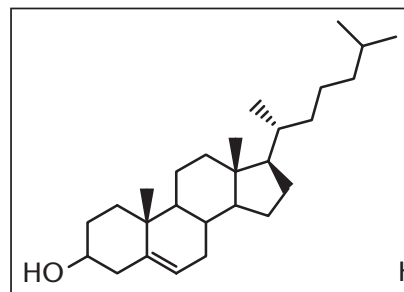

Cholestérol

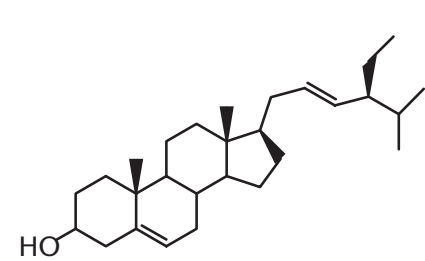

Stigmastérol

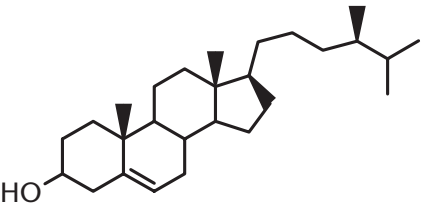

$\beta$-sitostérol

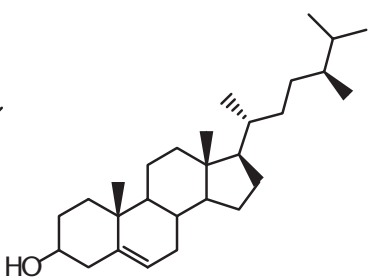

Campestérol
Figure 1. Structure du cholestérol et des principaux phytostérols.

nale du cholestérol en le remplaçant dans les micelles de sels biliaires [9, $16,17]$. Les phytostérols sont aussi étudiés pour leur action anticancéreuse [13], immunomodulatrice et anti-inflammatoire [18]. Les phytostérols possèdent également un pouvoir émulsifiant et pénétrant. Cette propriété est utilisée en cosmétique où ils sont souvent employés comme agents anti-dessiccateurs de l'épiderme et du cuir chevelu [7].

Comme pour les tocophérols [19], la teneur et composition en phytostérols varie en fonction de l'espèce végétale : soja, colza, tournesol, avoine et selon les variétés [10, 20]. La majorité des huiles végétales contient de 100 à $800 \mathrm{mg}$ de stérols. $100 \mathrm{~g}^{-1}$ d'huile. L'huile de tournesol présente majoritairement du $\beta$-sitostérol, puis du campestérol et du stigmastérol ce qui en fait une huile de profil original [21]. Par comparaison, le colza ne synthétise pas de stigmastérol et accumule du brassicastérol dont les effets sur la santé pourraient être négatifs et le soja accumule significativement moins de phytostérols [22]. Les différences génotypiques ont été observées chez le seigle [23], I'avoine [20], I'arachide [24] et le colza [25, 26]. L'effet environnemental affecte significativement la distribution des stérols, cependant l'effet du lieu de culture est beaucoup moins important que sur les tocophérols $[23,26]$ voire inexistant sur un essai réalisé sur de l'avoine [20]. Chez le soja, la concentration en phytostérols totaux augmente si la température est élevée durant la maturation de la graine [10]. On observe aussi une modification significative de la composition des phytostérols : augmentation du pourcentage en campestérol et diminution des teneurs en stigmastérol et en $\beta$-sitostérol [10].

Le tournesol est donc une espèce oléagineuse de grande culture dont le profil stérolique atypique est recherché pour des valorisations industrielles. Cependant, les teneurs totales en phytostérols sont inférieures à celles observées pour d'autres espèces oléagineuses (colza, maïs). La connaissance de la variabilité de teneur et composition en phytostérols des hybrides commerciaux est une première étape $d$ 'investigation pour envisager la faisabilité d'une sélection pour ce caractère. L'objectif de cette étude s'inscrit dans une démarche similaire à celle entreprise sur l'étude des tocophérols [19], à savoir suivre le remplissage de la graine en phytostérols totaux et individuels au cours de la maturation de l'akène chez des hybrides commerciaux pour deux années de culture (2002 et 2003). Ce travail permettra ainsi d'évaluer les influences génétiques et environnementales sur la teneur et la composition en phytostérols et de rechercher d'éventuelles phases critiques lors du remplissage.

\section{Matériel et méthodes}

\section{Matériel végétal}

En 2002, 4 variétés commerciales hybrides (Allstar RM, LG5420M, LG5660, Prodisol) ont été cultivées à Baziège (31). En 2003, onze variétés commerciales hybrides : neuf classiques (Alisson RM, Allstar RM, LG 5420M, LG5660, Melody, Parma, Tekny, Tellia, Prodisol) et deux variétés commerciales à haute teneur en acide oléique (Haut oléique) (Alisson $\mathrm{RMO}$, Aurasol) ont été mises en place sur trois lieux : Mondonville (31), St. Sauveur (31) et Caussade (81). Seuls les quatre hybrides de 2002 sont présents pendant les deux années d'essai (2002/2003).

Les variétés, l'itinéraire cultural, les prélèvements et la préparation des akènes sont décrits dans Ayerdi-Gotor et al. [19]. Comme pour l'étude sur les tocophérols, les prélèvements effectués sur le site de Baziège en 2002 et sur le site de Mondonville en 2003 ont été utilisés pour l'étude de la cinétique des teneurs et compositions en phytostérols au cours du remplissage des akènes (tableau 1). L'autofécondation a été assurée par empochage des capitules juste avant et pendant la période de la floraison. Après la floraison, les sacs ont été ouverts excepté sur le site de St Sauveur (31) où les capitules sont restés empochés jusqu'à la récolte. Pour les cinétiques, à chaque date de prélèvement, 3 à 5 capitules par parcelle ont été collectés. Pour des raisons d'homogénéité de stade de développement, seuls les trois cercles extérieurs de chaque capitule ont été égrenés. Les akènes des capitules sélectionnés ont été ensuite regroupés par parcelle, lyophilisés pendant 48 heures puis stockés à $-18^{\circ} \mathrm{C}$ jusqu'à l'analyse.

\section{Analyses}

\section{Extraction de l'huile}

L'huile des akènes a été extraite à I'hexane pendant 4 heures dans un appareil de Soxhlet [19]. Après extraction, I'huile de chaque échantillon a été récupérée et stockée dans des piluliers à $-18{ }^{\circ} \mathrm{C}$. Chaque échantillon a été analysé en double répétition.

\section{Analyse des phytostérols}

La détermination de la teneur en phytostérols des huiles brutes a été réalisée selon la norme ISO 12228 : 1999 [27]. La séparation complète des différents phytostérols a été obtenue par chromatographie en phase gazeuse (CPG) (GC 8000 - FISONS Instrument, Italie). Le principe de l'analyse des phytostérols est basé sur un isolement de l'insaponifiable puis une silylation de la fraction insaponifiable avant injection directe en chromatographie en phase gazeuse (CPG).

\section{Saponification}

Dans un ballon de $50 \mathrm{~mL}$, on introduit $250 \mathrm{mg}$ d'huile, $1 \mathrm{mg}$ de bétuline/mL d'acétone (bétuline : étalon interne - Sigma Aldrich, Saint Quentin Fallavier, France) et $5 \mathrm{~mL}$ d'une solution éthanolique d'hydro-

Tableau 1. Echelonnement des prélèvements réalisés en 2002 (4 hybrides) et en 2003 (11 hybrides).

\begin{tabular}{|llll|}
\hline Année & Lieu des essais & $\begin{array}{l}\text { Prélèvement } \\
\text { intermédiaire (JAF*) }\end{array}$ & $\begin{array}{l}\text { Prélèvement } \\
\text { final (JAF) }\end{array}$ \\
\hline 2002 & Baziège (31) & 20 à 60, tous les 5 jours & 60 \\
\hline 2003 & Caussade (82) & 35 & 77 \\
\cline { 2 - 4 } & Mondonville (31) & $20,30,40,53$ & 63 \\
\cline { 2 - 4 } & St. Sauveur (31) & I & 65 \\
\hline
\end{tabular}

* JAF : jours après floraison. 
xyde de potassium $(\mathrm{KOH} / 0,5 \mathrm{M})$. Le mélange est porté à ébullition sous reflux pendant 15 minutes. Immédiatement après l'arrêt de la saponification, $5 \mathrm{~mL}$ d'éthanol (qualité HPLC - SDS, France) sont ajoutés au mélange chaud.

\section{Extraction des stérols}

Pour isoler la fraction insaponifiable, $5 \mathrm{~mL}$ de la solution sont versés dans une colonne d'oxyde d'aluminium. Cette colonne en verre (diamètre : $30 \mathrm{~mm}$ ) est préalablement préparée et remplie de $10 \mathrm{~g}$ d'oxyde d'aluminium (neutre, $\mathrm{pH}=7,5,50-100 \mu \mathrm{m}$, PROLABO/SUBRA-France) hydratés avec de l'éthanol (Qualité HPLC, SDS, France). La substance résultant de la première élution n'est pas récupérée. Une modification de protocole a été effectuée en plaçant dans un entonnoir un filtre Whatmann (papier $n^{\circ} 41$ ) sur un deuxième ballon afin de retenir les particules d'alumine contenues dans l'éluat. L'insaponifiable est extrait par $5 \mathrm{~mL}$ d'éthanol puis par $30 \mathrm{~mL}$ d'éther diéthylique (Qualité HPLC, SDS, France). Les solvants sont évaporés sous vide (Rotavapor, Bioblock Scientific HS 40 HUBER, Heildolph). Le dépôt présent sur les parois est décollé avec $2 \mathrm{~mL}$ d'éther diéthylique et récupéré dans un tube à hémolyse. L'éther diéthylique est ensuite évaporé avec un flux continu d'azote (Air Liquide, France).

\section{Identification des stérols}

$100 \mu \mathrm{L}$ de réactif silylant (95 $\mu \mathrm{L}$ de $\mathrm{N}$-méthyle-N-(triméthylsilyl)-heptafluorobutyramide (Sigma Aldrich, Saint Quentin Fallavier, France) et $5 \mu \mathrm{L}$ de 1-méthyle imidazol (Sigma Aldrich, Saint Quentin Fallavier, France) sont ajoutés dans les tubes à hémolyse contenant les stérols isolés. Le tube est ensuite chauffé pendant 15 min à $105^{\circ} \mathrm{C}$ dans un bain d'huile préchauffé.

$0,5 \mu \mathrm{L}$ ont été injectés manuellement avec une micro-seringue (ou $1 \mu \mathrm{L}$ par le passeur automatique) sur une colonne capillaire en silice fondue SAC-5 (30 cm $\times 0,25 \mathrm{~mm} \times 0,25 \mu \mathrm{m}$, Cluzeau, Puteaux La Défense, France), I'injecteur est à $320^{\circ} \mathrm{C}$, et l'azote (gaz vecteur) à $1 \mathrm{~mL} / \mathrm{min}$ avec une pression de $130 \mathrm{kPa}$ (Air Liquide, France). Un gradient de température de 240 à $320{ }^{\circ} \mathrm{C}$ avec une augmentation de $4{ }^{\circ} \mathrm{C} /$ min est appliqué, la température finale est ensuite laissée constante pendant 10 min. Les différents phytostérols sont quantifiés par un détecteur à ionisation de flamme à $310{ }^{\circ} \mathrm{C}$ avec un débit d'hydrogène de $30 \mathrm{~mL} / \mathrm{min}$ et un débit d'air de $300 \mathrm{~mL} / \mathrm{min}$. Le signal a été intégré avec l'appareil Data Jet Integrator (Thermo Finnigan, Courtaboeuf, France).

Chaque phytostérol est identifié à partir de son temps de rétention défini à l'aide de mélanges d'étalons standards ( $\beta$-sitostérol, stigmasterol et campestérol (Sigma Aldrich, Saint Quentin Fallavier, France)).

\section{Calibration interne - Quantification des phytostérols}

Pour quantifier les stérols présents dans l'échantillon, les temps de rétention relatifs (TRR) ont été obtenus en divisant le temps de rétention (TR) du stérol concerné par le TR de la bétuline. Dans cette méthode, on considère que les facteurs de réponse de tous les stérols et de la bétuline sont égaux. La quantification a été calculée à partir des aires des pics pour les différents phytostérols comparée à l'aire du pic correspondant à l'étalon interne (bétuline).

\section{Analyse des phytostérols avant 20 jours après floraison}

Sur des stades très jeunes de la plante (avant 20 jours après floraison JAF), I'insuffisance d'huile extraite n'a pas permis de réaliser le dosage des phytostérols par la méthode ISO 12228 : 1999. L'extraction a été réalisée par une méthode directe sur broyat de graines basée sur la méthode normée NF 5508 [28] et réalisée par le Laboratoire de chimie agro-industrielle de I'ENSIACET. Les résultats par échantillon correspondent à la moyenne de 4 essais; et ils sont exprimés en mg stérols pour $100 \mathrm{~g}$ de matière sèche d'akènes. La teneur en huile a été déterminée sur un échantillon indépendant.

\section{Traitement des données}

Les analyses statistiques (corrélations et analyses de la variance (ANOVA) à deux facteurs) ont été réalisées sous Microsoft Excel pour Windows ${ }^{\circledR}$ 97 et sous SPSS 11.0 pour Windows ${ }^{\circledR}$ (Paris, France).

\section{Résultats}

Les chromatogrammes obtenus (figure 2) montrent une séparation efficace des différents phytostérols et de l'étalon interne. Ainsi pour l'intégration des pics, les temps de rétention relatifs (TRR) calculés par rapport à la bétuline sont : campestérol $(0,79)$; stigmastérol $(0,81)$; $\Delta^{5}$-campestérol $(0,83) ; \beta$-sitostérol $(0,85) ; \Delta^{5}$-avénastérol $(0,86)$; $\Delta^{7}$-stigmastérol $(0,94)$ et $\Delta^{7}$-avénastérol $(0,96)$. Les teneurs en phytostérols sont exprimées en $\mathrm{mg} \cdot 100 \mathrm{~g}^{-1} \mathrm{~d}^{\prime}$ huile ou en $\mathrm{mg}$ par $100 \mathrm{~g}$ de matière sèche $\mathrm{d}^{\prime}$ akènes $\left(\mathrm{mg} \cdot 100 \mathrm{~g}^{-1} \mathrm{MS}\right)$.

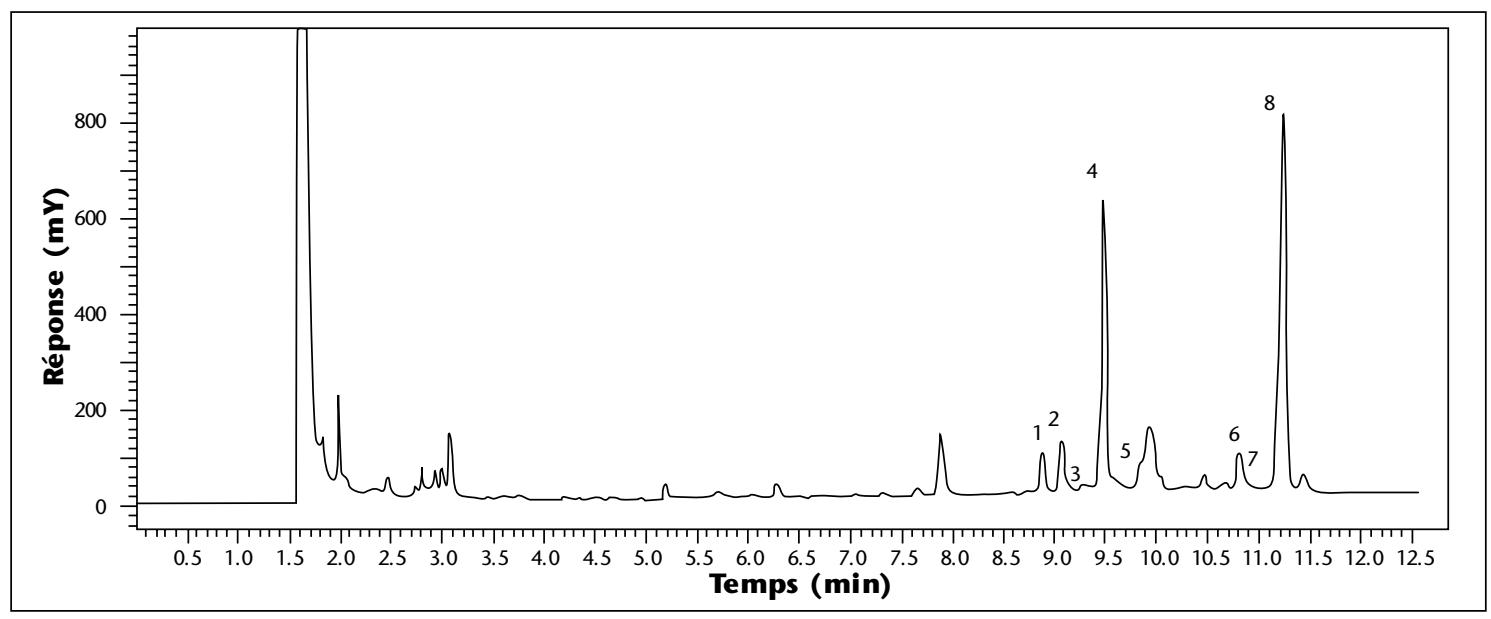

Figure 2. Composition en stérols d'une huile de tournesol obtenue par chromatographie en phase gazeuse sur colonne capillaire en silice fondue SAC-5 (Cluzeau, Paris) (Pics : 1 : campestérol, $2:$ stigmastérol ; $3: \Delta^{7}$-campestérol ; $4: \beta$-sitostérol ; $5: \Delta^{5}$-avénastérol; $6: \Delta^{7}$-stigmastérol; $7: \Delta^{7}$-avénastérol; $8:$ bétuline). 
Effets du lieu de culture et du génotype sur la teneur en phytostérols totaux à maturité

La teneur en phytostérols totaux à maturité a été analysée dans I'huile de 11 hybrides cultivés sur trois lieux en 2003 (tableau 2). Elle varie entre 329 et $595 \mathrm{mg} \cdot 100 \mathrm{~g}^{-1} \mathrm{~d}^{\prime}$ huile et entre 142 et $268 \mathrm{mg} \cdot 100 \mathrm{~g}^{-1} \mathrm{de}$ matière sèche. Tous comme les résultats obtenus sur les tocophérols, la différence significative observée entre les essais est probablement liée à leurs conditions de réalisation. Sur le site de Saint Jory, les capitules sont restés empochés jusqu'à la récolte, ce qui a engendré un stress thermique supplémentaire dû au sac. L'effet de l'environnement, essentiellement de la température, semble être un facteur clé dans la teneur en phytostérols. Ces résultats semblent indiquer que les températures élevées induisent une augmentation de la concentration de l'huile en phytostérols. L'analyse de variance (ANOVA) montre que les effets génétiques et environnementaux affectent les teneurs en phytostérols (tableau 3) mais ces variations sont moindres par rapport à celles observées sur les teneurs en tocophérols [19]. L'interaction génotype $\times$ lieu présente une part de la variance totale similaire à la variation génotypique.

Tableau 2. Moyennes des teneurs en phytostérols totaux (mg.100 $\mathrm{g}^{-1} \mathrm{~d}^{\prime}$ huile). Les erreurs standard sont respectivement 19, 13 et 43 pour les moyennes des variétés ( $n=6$ échantillons par variété), des lieux ( $n=20$ échantillons par lieu) et des variétés par lieu ( $n=2$ répétitions).

\begin{tabular}{|llccc|}
\hline Variétés & $\begin{array}{l}\text { Moyenne variétés } \\
\mathbf{2 0 0 3}\end{array}$ & $\begin{array}{l}\text { Mondonville } \\
\mathbf{2 0 0 3}\end{array}$ & $\begin{array}{l}\text { Caussade } \\
\mathbf{2 0 0 3}\end{array}$ & $\begin{array}{l}\text { Saint Jory } \\
\mathbf{2 0 0 3}\end{array}$ \\
\hline Alisson & $442^{\mathrm{ab}}$ & 419 & 407 & 499 \\
\hline Alisson RMO & $483^{\mathrm{ab}}$ & 382 & 418 & 595 \\
\hline AllStar & $437^{\mathrm{ab}}$ & 398 & 378 & 535 \\
\hline Aurasol & $423^{\mathrm{ab}}$ & 346 & 334 & 589 \\
\hline LG5420 & $403^{\mathrm{a}}$ & 371 & 438 & 401 \\
\hline LG5660 & $432^{\mathrm{ab}}$ & 474 & 369 & 454 \\
\hline Melody & $443^{\mathrm{ab}}$ & 401 & 384 & 546 \\
\hline Parma & $420^{\mathrm{ab}}$ & 486 & 329 & 476 \\
\hline Prodisol & $469^{\mathrm{ab}}$ & 510 & 355 & 543 \\
\hline Tellia & $530^{\mathrm{b}}$ & 561 & 462 & 567 \\
\hline $\begin{array}{l}\text { Moyennes } \\
\text { lieux }\end{array}$ & & $436^{\mathrm{b}}$ & $387^{\mathrm{a}}$ & $520^{\mathrm{c}}$ \\
\hline
\end{tabular}

Tableau 3. Résultats de l'analyse de variance de 11 hybrides de tournesol cultivés sur 3 lieux (Caussade (81), Mondonville (31) et St Jory (31)).

\begin{tabular}{|c|c|c|c|c|}
\hline & \multirow[t]{2}{*}{ dl } & \multicolumn{3}{|c|}{$\begin{array}{l}\text { Phytostérols totaux } \\
\text { (mg } .100 \mathrm{~g}^{-1} \mathrm{~d}^{\prime} \text { huile) }\end{array}$} \\
\hline & & SC & MC & $\mathbf{F}$ \\
\hline Lieu & 2 & 374167 & 187083 & $55,5^{*}$ \\
\hline Génotype & 9 & 126481 & 14053 & $4,2^{*}$ \\
\hline$L \times G$ & 18 & 217014 & 12056 & $3,6^{*}$ \\
\hline Erreur & 83 & 280027 & 3374 & \\
\hline
\end{tabular}

dl. degré de liberté - SC. Somme des carrés - MC. Moyenne des carrés - F. Test statistique ${ }^{*} \mathrm{p}<0,001$.
Évolution de la teneur en phytostérols totaux au cours de la maturation de l'akène

Par souci de clarté, les cinétiques d'accumulation présentées graphiquement ici concernent uniquement les variétés Prodisol et Allstar, représentatives de l'évolution des autres variétés étudiées.

La fraction lipidique commence à s'accumuler dès le $10^{\mathrm{e}}$ jours après floraison (JAF) et atteint rapidement un plateau vers le $25^{\mathrm{e}} \mathrm{JAF}$, seuil audelà duquel le pourcentage d'huile se stabilise jusqu'à la maturité de l'akène (figure 3). Les teneurs observées en 2003 sont cependant nettement plus faibles.

La teneur en phytostérols totaux exprimée par rapport à l'huile (mg·100 $\mathrm{g}^{-1} \mathrm{~d}^{\prime}$ huile, figure 4), ne semble pas montrer d'accumulation, mais une teneur finale établie dès 20 à 30 JAF après une forte diminution de la teneur initiale. En fait, cette diminution coïncide avec le début du remplissage en huile de l'akène : elle traduit donc un effet de dilution des phytostérols issus de la phase lipidique des tissus de l'embryon. Par la suite, la teneur en phytostérols reste constante, qu'elle soit exprimée par rapport à la matière sèche (figure 5) ou par rapport à I'huile : ceci traduit donc une synthèse active de phytostérols synchrone à celle des acides gras.

En 2003 les teneurs exprimées par rapport à la MS sont similaires à celles de 2002 sur les stades précoces. Elles apparaissent cependant nettement plus faibles au cours de la maturation : ceci peut s'expliquer par l'effet année sur la teneur en huile, comme le montre la figure 3. On constate qu'avant $40 \mathrm{JAF}$, la concentration en phytostérols dans I'huile reste plus élevée en 2003. Ceci pourrait être lié à l'épisode caniculaire du début du mois d'août.

L'analyse de variance (tableau 4) réalisée sur les 4 hybrides: Prodisol, Allstar RM, LG5420 et LG5660, sur l'ensemble des prélèvements et sur les deux années 2002 et 2003, montre qu'il n'y a pas de différence de génotype mais qu'un effet année peut être mis en évidence sans être aussi marqué que celui observé sur les teneurs en tocophérols [19].

\section{Évolution des teneurs en phytostérols individuels au cours de la maturation de l'akène}

L'ensemble des phytostérols ( $\beta$-sitostérol, stigmastérol, campestérol) présente une cinétique d'accumulation similaire à celle des phytostérols totaux (mg.100 g $\mathrm{g}^{-1} \mathrm{~d}$ 'huile). Le $\beta$-sitostérol est en quantité majoritaire

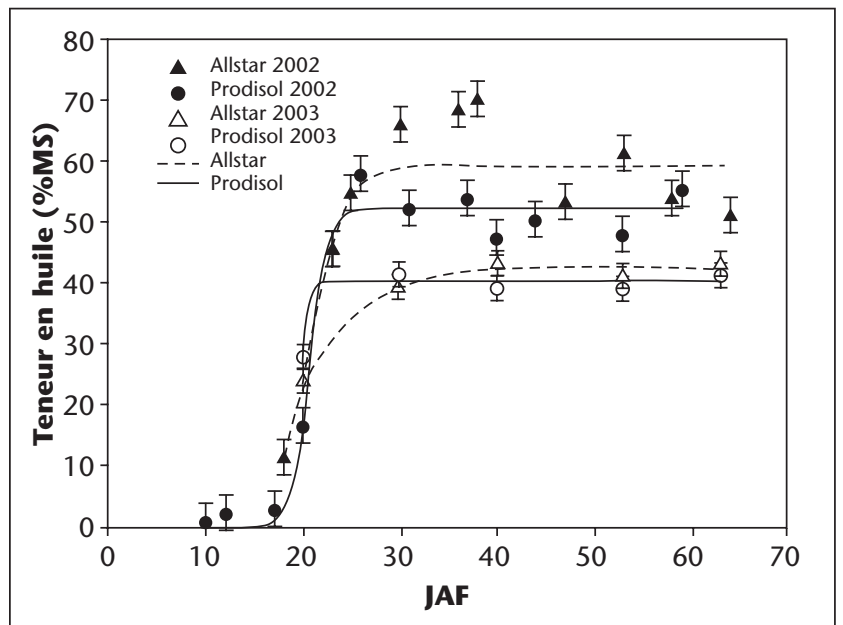

Figure 3. Accumulation de l'huile (\% de la matière sèche) de 10 jours après floraison (JAF) à maturité pour les variétés Prodisol et Allstar RM sur les essais réalisés en plein champ à Baziège en 2002 et à Mondonville en 2003. Chaque point = moyenne de 3 répétions analytiques. 


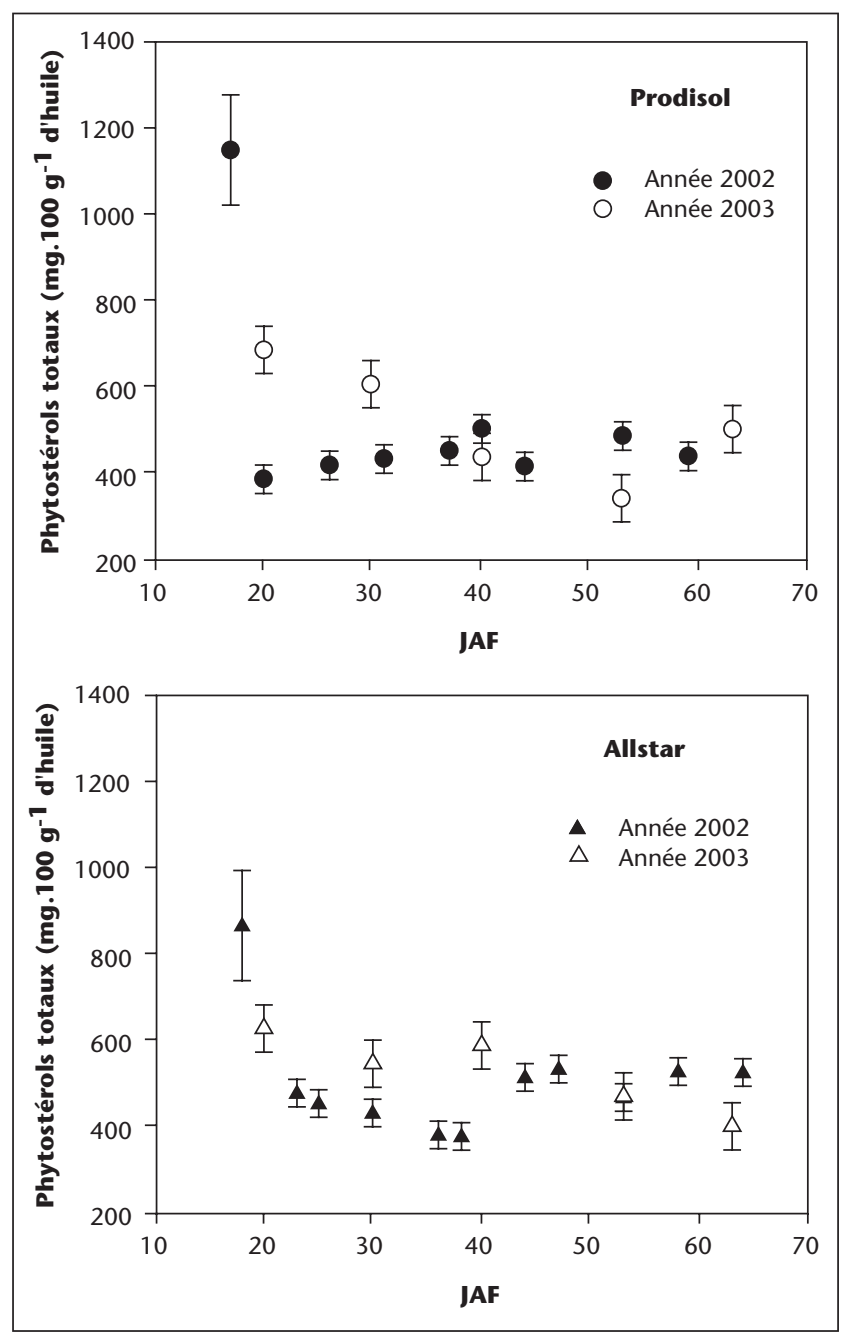

Figure 4. Accumulation des phytostérols exprimée en $\mathrm{mg} .100 \mathrm{~g}^{-1} \mathrm{~d}$ 'huile de 10 jours après floraison (JAF) à maturité pour les variétés Prodisol et Allstar RM sur les essais réalisés en plein champ à Baziège en 2002 et à Mondonville en 2003. Chaque point $=$ moyenne de 3 répétions analytiques.

(environ $60 \%$ ) ; les autres phytostérols représentent chacun $10 \%$ (ou moins) des phytostérols totaux. Contrairement à ce qui a été observé pour les tocophérols, la composition en phytostérols (en mg par $100 \mathrm{~g}$ $d^{\prime}$ 'huile) semble relativement constante dès 20 JAF (tableau 5). Cependant, le $\beta$-sitostérol semble disparaître au cours de la maturation pour certaines variétés telles que LG 5420 et LG5660, ce qui entraînerait la décroissance observée à maturité.

\section{Discussion Conclusion}

Cette étude de la teneur en phytostérols à maturité de différents hybrides commerciaux montre que les différences variétales sont moins importantes pour la teneur en phytostérols que pour les tocophérols. Un classement des hybrides par rapport à leur teneur en phytostérols dans les akènes à maturité est beaucoup plus difficile à réaliser que pour les tocophérols [19]. La variété Tellia présente un potentiel élevé en phytostérols tandis que la variété Melody se classe significativement parmi les plus faibles.
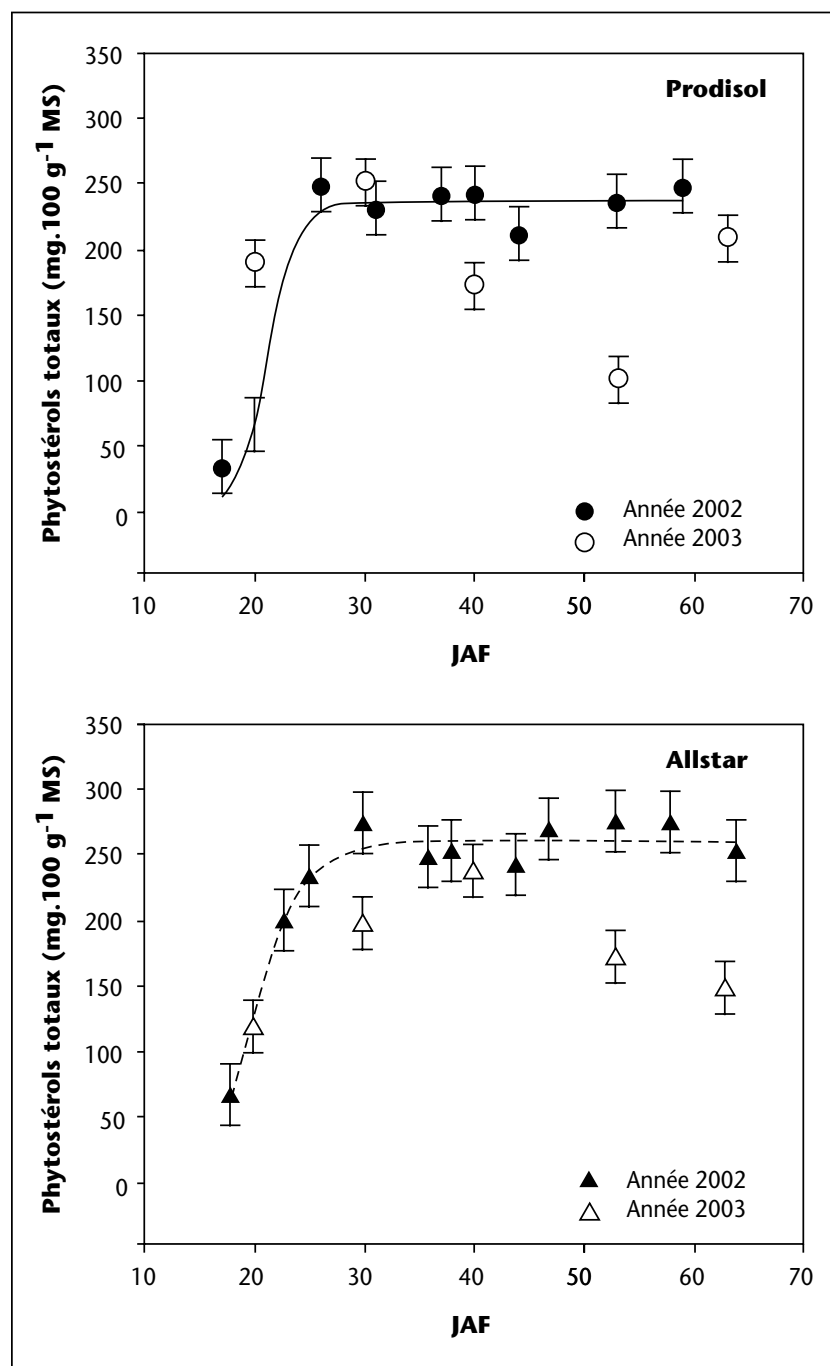

Figure 5. Accumulation des phytostérols exprimée en $\mathrm{mg} 100 \mathrm{~g}^{-1}$ de MS de 10 jours après floraison (JAF) à maturité pour les variétés Prodisol et Allstar RM sur les essais réalisés en plein champ à Baziège en 2002 et à Mondonville en 2003. Chaque point $=$ moyenne de 3 répétions analytiques.

Tableau 4. Résultats de l'analyse de variance en moyenne des carrés des tocophérols et phytostérols totaux de 4 hybrides (Allstar, Prodisol, LG5420 et LG 5660) cultivés en 2002 et 2003 et 5 dates de prélèvement à 20,30,40, 55 et 65 jours après floraison (JAF).

\begin{tabular}{|c|c|c|c|}
\hline Facteurs & d.I. & $\begin{array}{l}\text { Tocophérols totaux } \\
\left(\mathbf{m g} \cdot \mathbf{k g}^{-1} \text { huile) }\right.\end{array}$ & $\begin{array}{l}\text { Phytostérols totaux } \\
\text { ( } \mathrm{mg} \cdot 100 \mathrm{~g}^{-1} \text { huile) }\end{array}$ \\
\hline JAF & 1 & $62135^{*}$ & $128441^{* * *}$ \\
\hline Génotype & 3 & $114230^{* * *}$ & 3606 \\
\hline Année & 1 & $278790^{* *}$ & $26836^{*}$ \\
\hline Génotype $\times$ Année & 3 & 9463 & 717 \\
\hline Erreur & 53 & 9306 & 5947 \\
\hline
\end{tabular}

JAF : jours après floraison; d.I. Degré liberté ; Test $\mathrm{F}:{ }^{*} \mathrm{p}<0,05 ;{ }^{* *} \mathrm{p}<0,01$; $p<0,001$. 
Tableau 5. Teneurs en campésterol, stigmastérol, $\beta$-sitostérol et phytostérols totaux, exprimées en mg. $100 \mathrm{~g}^{-1}$ d'huile, de deux variétés à 5 stades $20,30,40,55$ et 65 jours après floraison (JAF) de la graine pour deux années de culture 2002 et 2003.

\begin{tabular}{|c|c|c|c|c|c|c|c|}
\hline \multirow[t]{2}{*}{ Variétés } & \multirow[t]{2}{*}{ Phytostérols } & \multirow[t]{2}{*}{ Année } & \multicolumn{5}{|c|}{ Jours après floraison (JAF) } \\
\hline & & & 20 & 30 & 40 & 55 & 65 \\
\hline \multirow[t]{7}{*}{ Allstar RM } & \multirow[t]{2}{*}{ Campestérol } & 2002 & 45,50 & 37,00 & 31,00 & 45,40 & 45,00 \\
\hline & & 2003 & 53,43 & 60,19 & 52,18 & 41,26 & 31,13 \\
\hline & \multirow[t]{2}{*}{ Stigmastérol } & 2002 & 60,00 & 44,50 & 39,00 & 56,00 & 56,50 \\
\hline & & 2003 & 100,83 & 66,16 & 57,43 & 53,81 & 36,29 \\
\hline & \multirow[t]{2}{*}{$\beta$-sitostérol } & 2002 & 297,00 & 278,00 & 243,00 & 338,00 & 332,00 \\
\hline & & 2003 & 386,54 & 243,00 & 363,37 & 302,50 & 295,22 \\
\hline & Phytostérols totaux & 2002 & 472,50 & 426,50 & 376,50 & 527,00 & 516,00 \\
\hline \multirow[t]{9}{*}{ Prodisol } & \multirow[t]{3}{*}{ Campestérol } & 2003 & 624,72 & 591,04 & 533,25 & 470,74 & 398,31 \\
\hline & & 2002 & 32,33 & 40,33 & 43,00 & 44,67 & 40,67 \\
\hline & & 2003 & 91,05 & 61,02 & 46,72 & 21,85 & 41,55 \\
\hline & \multirow[t]{2}{*}{ Stigmastérol } & 2002 & 54,33 & 48,67 & 47,67 & 50,67 & 50,00 \\
\hline & & 2003 & 84,68 & 61,22 & 49,83 & 24,99 & 47,17 \\
\hline & \multirow[t]{2}{*}{$\beta$-sitostérol } & 2002 & 268,00 & 289,33 & 270,67 & 313,67 & 293,67 \\
\hline & & 2003 & 333,82 & 277,60 & 310,01 & 147,69 & 326,02 \\
\hline & \multirow[t]{2}{*}{ Phytostérols totaux } & 2002 & 391,00 & 425,00 & 460,33 & 492,00 & 444,33 \\
\hline & & 2003 & 621,92 & 487,96 & 470,96 & 231,43 & 458,30 \\
\hline
\end{tabular}

L'effet de l'environnement et plus particulièrement de la température interviendrait sur la teneur en phytostérols totaux. L'effet des fortes températures observées en 2003 et un stress thermique supplémentaire au niveau du capitule favorisent des teneurs plus élevées en phytostérols totaux. Ces résultats corroborent ceux décrits par Vlahakis et Hazebroek [10] chez le tournesol. Cependant, ces effets ne sont pas comparables à ceux observés sur les tocophérols. De fortes températures durant le remplissage de l'akène limiteraient l'accumulation des tocophérols car ils pourraient être utilisés pour protéger les acides gras accumulés de dégradation subie lors du stress oxydatifs induit par les températures élevées $[19,29]$. La température semble être un facteur clé dans la teneur en phytostérols et des investigations supplémentaires devront être menées dans des conditions contrôlées afin d'affirmer l'importance de ce facteur. En effet, si l'effet de la chaleur induisait des teneurs plus élevées alors des semis plus précoces et à fortiori des récoltes plus précoces dans des régions du Sud de l'Europe conduiraient à des variétés à plus haute teneur en phytostérols.

Après avoir étudié la variabilité des phytostérols à maturité, il nous a semblé nécessaire, dans une seconde phase, d'apporter des connaissances sur le remplissage de l'akène en phytostérols. La cinétique d'accumulation des phytostérols totaux montre qu'ils sont présents dans tous les stades de développement de la graine. L'accumulation en phytostérols dans l'akène comporte deux périodes. Pendant la première phase, la teneur en phytostérols totaux diminue fortement pour atteindre une teneur finale qui s'établit dès 20 à 30 jours après floraison (JAF) selon le génotype. La diminution pourrait correspondre au début de l'accumulation de l'huile qui entraînerait alors une dilution des phytostérols dans les tissus de l'embryon. Dans un second temps, la stabilisation observée indique que l'accumulation active des phytostérols a commencé et qu'elle est synchrone à celle des acides gras. L'étude du remplissage des tocophérols dans des akènes de tournesol a mis en évidence que la phase d'accumulation active des tocophérols coïncidait avec celle des acides gras et de la matière sèche. L'étude de la cinétique montre une augmentation de la teneur en $\alpha$-tocophérol jusqu'à 25 JAF puis une stabilisation tandis que les autres tocophérols ( $\beta$ et $\gamma$ ) diminuaient progressivement. Pour les phytostérols, nous ne notons pas de variation significative du profil de composition.

On constate qu'en 2003 avant 40 JAF, période de la canicule du début du mois d'août, la teneur en phytostérols dans I'huile reste plus élevée. II a été montré que les phytostérols ont un rôle dans I'adaptation des plantes aux fortes températures [30, 31]. Par contre, de fortes températures durant le remplissage de l'akène limiteraient l'accumulation des tocophérols afin de préserver la semence du stress oxydatif, de manière similaire aux mécanismes mis en jeu au niveau de la feuille [31]. Les travaux de Harker et al. [32] sur des graines de colza et de tabac montrent des résultats similaires.

En conclusion, cette étude montre que la variabilité de la teneur en phytostérols dans I'huile de tournesol est due au génotype et à un effet environnemental amplifié par de fortes températures pendant la maturation de la graine. Cependant, la température apparaît comme un des facteurs environnementaux le plus déterminant sur les teneurs finales en tocophérols et en phytostérols dans I'huile. Au regard des résultats, il s'avère qu'augmenter les teneurs en tocophérols dans des akènes de tournesol sera possible par la sélection variétale tandis que l'enrichissement en phytostérols sera plus limité. Les conditions culturales et la date de prélèvement seront des facteurs clés sur les teneurs en phytostérols. II serait nécessaire d'établir plus précisément l'effet de la température sur la teneur en phytostérols finale en effectuant un essai avec des conditions environnementales contrôlées, mais également de prospecter sur un potentiel de variabilité génétique plus riche. 


\section{RÉFÉRENCES}

1. Merrien A, Morice J, Pouzet A, Morin O, Sultana C. Graines oléagineuses des climats tempérés et leurs huiles. In : Karleskind A, ed. Manuel des Corps Gras. Paris : Tec\&Doc, 1992 : 116-64.

2. Lagravere T, Kleiber D, Dayde J. Conduites culturales et performances agronomiques du tournesol oléique : réalités et perspectives. OCL $1998 ; 5$ : 477-86.

3. Bramley PM, Elmafda I, Kafatos A, et al. Vitamin E. / Sc Food Agr 2000; 80 : 913-38.

4. Ballerini D. Les biocarburants : État de lieux, perspectives et enjeux du développement. Paris : IFP Publications, 2006 (1st).

5. Cervero JM, Coca J, Luque S. Production of biodiesel from vegetable oils. Grasas y Aceites $2008 ; 59: 76-83$.

6. Schneider MP. Plant-oil-based lubricants and hydraulic fluids. / SC Food Agr $2006 ; 86: 1769-80$.

7. Folmer BM. Sterol surfactants : from synthesis to applications. Adv Coll Interface Sc $2003 ; 103$ : 99-119.

8. Dutta P, Normen L. Capillary column gas- liquid chromatographic separation of delta 5-unsaturated and saturated phytosterol. / Chromatogr $A$ $1998 ; 816: 177-84$.

9. Normen L, Dutta P, Lia A, Andersson H. Soy sterol esters and beta-sitostanol ester as inhibitors of cholesterol absorption in human small bowel. Am / Clin Nutr $2000 ; 71: 908-13$.

10. Vlahakis $C$, Hazebroek J. Phytosterol accumulation in canola, sunflower, and soybean oils : Effects of genetics, planting location, and temperature. / Am Oil Chem Soc $2000 ; 77: 49-53$.

11. Lindsey K, Pullen ML, Topping JF. Importance of plant sterols in pattern formation and hormone signalling. Trends Plant Sc $2003 ; 8$ : 521-5.

12. Schaller $\mathrm{H}$. The role of sterols in plant growth and development. Prog Lip Res $2003 ; 42$ : 163-75.

13. Awad AB, Fink CS. Phytosterols as anticancer dietary components: Evidence and mechanism of action. / Nutr $2000 ; 130$ : 2127-30.

14. Orzechowski A, Ostaszewski P, Jank M, BERWID SJ. Bioactive substances of plant origin in food - impact on genomics. Reprod Nutr Dev 2002; 42 : 461-77.

15. St-Onge MP, Jones PJH. Phytosterols and human lipid metabolism : Efficacy, safety, and novel foods. Lipids $2003 ; 38: 367-75$.

16. Trautwein EA, Duchateau G, Lin YG, Mel'nikov SM, Molhuizen HOF, Ntanios FY. Proposed mechanisms of cholesterol-lowering action of plant sterols. Eur / Lip Sc Technol $2003 ; 105: 171-85$.

17. Ikeda I, Tanaka K, Sugano M, Vahouny GV, Gallo LL. Inhibition of cholesterol absorption in rats by plant sterols. J Lip Res 1988 ; 29 : 1573-82.

18. Bouic PJD, Clark A, Lamprecht J, et al. The effects of $\beta$-sitosterol (BSS) and $\beta$ sitosterol glucoside (BSSG) mixture on selected immune parameters of marathon runners: Inhibition of post marathon immune suppression and inflammation. Int / Sports Med $1999 ; 20: 258-62$.
19. Ayerdi Gotor A, Berger M, Labalette F, Centis S, Dayde J, Calmon A. Etude de la variation des teneurs et compositions des composés mineurs dans I'huile de tournesol au cours du développement du capitule : Partie I Tocophérols. OCL $2006 ; 13: 206-12$.

20. Määttä K, Lampi AM, Petterson J, et al. Phytosterol content in seven oat cultivars grown at three locations in Sweden. / Sc Food Agr 1999; 79 : 1021-7.

21. EC, 1995. European Parliament and Council Directive No 95/2/EC. Foods Additives other than colours and sweeteners. pp. 1-40.

22. Ayerdi-Gotor A. Etude des variations des teneurs et de la variabilité des compositions en tocophérols et en phytostérols dans les akènes et l'huile de tournesol (Helianthus annuus L.). Thèse de doctorat, Université de Toulouse-INPT 2009; 192pp.

23. Zangenberg M, Hansen HB, Jorgensen JR, Hellgren LI. Cultivar and yearto-year variation of phytosterol content in rye (Secale cereale L.). J Agr. Food Chem $2004 ; 52$ : 2593-7.

24. Jonnala RS, Dunford NT, Dashiell KE. Tocopherol, phytosterol and phospholipid compositions of new high oleic peanut cultivars. J Food Comp Anal $2006 ; 19: 601-5$.

25. Beermann C, Green A, Mobius M, Schmitt J], Boehm G. Lipid class separation by HPLC combined with GC FA analysis: Comparison of seed lipid compositions from different Brassica napus L. varieties. J Am Oil Chem Soc $2003 ; 80: 747-53$.

26. Hamama AA, Bhardwaj HL, Starner DE. Genotype and growing location effects on phytosterols in canola oil. J Am Oil Chem Soc $2003 ; 80$ : 1121-6.

27. NF EN ISO 12228, 1999. Association française de normalisation, 1999. Norme européenne, de mai 1999; Norme française T 60-258: Corps gras d'origines animale et végétale -Détermination de la teneur en stérols individuels et totaux - Méthode par chromatographie en phase gazeuse. AFNOR. Paris. 18.

28. Roche, J. Composition de la graine de tournesol (Helianthus annuus L.) sous l'effet conjugué des contraintes agri-environnementales et des potentiels variétaux. Thèse $n^{\circ} 2225$ 2005; Sciences des Procédés Spécialité : Sciences des Agroressources. Institut National Polytechnique de Toulouse. Toulouse. p 305.

29. Munne-Bosch S, Alegre L. Plant aging increases oxidative stress in chloroplasts. Planta $2002 ; 214: 608-15$.

30. Palta JP, Whitaker BD, Weiss LS. Plasma membrane lipids associated with genetic variability in freezing tolerance and cold acclimation of Solanum species. Plant Physiology $1993 ; 103$ : 793-803.

31. Buchanan BG, Jones RL. In : Membrane structure and membranous organelles. Biochemistry \& molecular biology of plants. Maryland, US : Am Soc Plant Physiol, 2000 : 850-907.

32. Harker M, Hellyer A, Clayton JC. Co-ordinate regulation of sterol biosynthesis enzyme activity during accumulation of sterols in developing rape and tobacco seeds. Planta $2003 ; 216: 707-15$. 